\title{
Transmittancy of Commercial Sugar Liquors: Dependence on Concentration of Total Solids
}

\author{
V. R. Deitz, N. L. Pennington, and H. L. Hoffman, Jr. ${ }^{2}$
}

\begin{abstract}
The dependence of the transmittancy of a commercial sugar liquor on the concentration of total solids does not in general follow Beer's law. This behavior is attributed to the presence of appreciable light-scattering material in the solution. A linear dependence has been found to relate the concentration with the reciprocal of $a_{c}^{*}$, the latter being; defined as equal to $\left(-\log T_{s}\right) / b c$, where $T_{s}$ is the transmittancy, $b$ the cell length, and $c$ the concentration of total solids. The intercept at $c=0$ in a plot of $1 / a_{c}^{*}$ versus $c$ is equal in magnitude to the limiting slope of the Beer's law plot.
\end{abstract}

\section{Introduction}

The loss in flux by absorption from a beam of radiant energy passing through a solution has long been used for the determination of dissolved substances. The scattering of radiant energy is known to interfere with the optical measurements, but since the nature of the interference is not well understood, it has generally been easier to minimize the scattering than to correct for it. There are, however, many commercially important solutions, sugar liquors for example, in which both absorption and scattering are appreciable. In order to characterize the optical properties of such solutions, the influence of the concentration of total solids upon the spectral transmittancy of these solutions has been studied.

The concentration of the nonsucrose solids in commercial sugar liquors is small compared to that of the total solids and, furthermore, is not subject to any convenient direct measurement. In addition to sucrose and water, the liquor contains dissolved and suspended materials of variable composition $[1,2]^{3}$ that absorb and scatter light to different extents. Therefore, it is common procedure for sugar technologists to describe the concentration of commercial sugar liquors by the concentration of total solids, realizing always that the nonsucrose solids compose only a very small fraction of the total and that this may be different for each commercial product. Thus, it is only possible at present to make a transmittancy measurement corresponding to a relative concentration of nonsucrose solids, it always being tacitly assumed that the light-absorbing and light-scattering materials are diluted in proportion to the total solids.

It should be noted that all transmission measurements reported in this paper were made on a model D. U. Beckman Spectrophotometer. Because of the presence of light-scattering materials, the values of transmittancy will be a function of the geometrical arrangement of the source, test medium, and the detector. This arrangement varies in instruments

\footnotetext{
1 This investigation was sponsored as a joint research project undertaken by the Bone Char Research Project, Inc., and the National Bureau of Standards. ${ }^{2}$ Research Associate at the National Bureau of Standards, representing the Bone Char Research Project, Inc.

3 Figures in brackets indicate the literature references at the end of this paper.
}

of different manufacture. Different values may also be obtained because of variations in the relative amounts of absorption and scattering material that may be present in particular commercial sugar liquors from different sources.

\section{Definition of Optical Terms}

The optical nomenclature used in this paper is that given by K. S. Gibson in 1949 [3]. Absorbancy is defined as

$$
A_{s}=-\log _{10} \frac{T_{\text {soln. }}}{T_{\text {solv. }}}=-\log _{10} T_{s}
$$

where $T_{\text {soln. }}$ and $T_{\text {solv. }}$ are the transmittance of the solution and solvent, respectively, and $T_{s}$ is the transmittancy. Transmittance is defined as the ratio of the transmitted flux to the incident flux of the spectral energy being used. The definition of absorbancy differs from that previously used by Bates and Associates [4] in 1942, where absorbancy was defined as $\left[1-T_{s}\right], T_{s}$ being the transmittancy of the solution.

The absorbancy index is defined as

$$
a_{s}=\frac{A_{s}}{b c}
$$

which is equivalent to the specific absorptive index used by Bates and Associates and denoted by them as $-\log t$. In eq (2), $b$ is the cell length, and $c$ is the concentration of the solute or constituent of interest. When applied to commercial sugars, $c$ is the concentration of total solids in grams per milliliter of solution. Total solids comprise the sugars and nonsugars, including the colored material.

The treatment of Bates or of Gibson is strictly correct only when no radiant energy is scattered from the initial direction of propagation. Equation (2) is a formulation of the Lambert-Beer law [5] and has long been used by sugar technologists to determine the value of the absorbancy index, $a_{s}$, from observations on "optically clear" sugar solutions. The term "optically clear" has been used to indicate that the solutions did not appreciably scatter light. It is now recognized that the sucrose molecule itself 
scatters light to a measurable extent [6]. The scattering in intensively purified sucrose solutions is small and is of the magnitude ascribed to molecular scattering. For these solutions the treatment of Bates or Gibson is valid, but it is not valid for the general case of commercial sugar liquors. The absorbancy index is constant in general only for nonscattering solutions. The present work shows that this is not the case for commercial sugar liquors.

In order to avoid ambiguity, the term "absorbancy index" will not be used when both absorption and scattering decrease the intensity of light. Instead, the term "attenuation index" will be used. "The attenuation index is an unknown function of absorption and scattering and cannot be readily analyzed into its components: (1) the molecular absorption due to dissolved substances, (2) the absorption by the suspended particles, (3) the scattering by the suspended particles, and (4) the scattering by the molecules of the solution (negligibly small in the case of sucrose solutions). The attenuation index, $a_{c}^{*}$, however, can be defined in terms of the observed transmittancy, the cell length and the concentration. of total sugar solids as follows:

$$
a_{c}^{*}=\frac{-\log T_{s}}{b c}
$$

In the absence of scattering, the attenuation index is equal to the absorbancy index $\left(a_{s}\right)$, which is independent of the concentration but dependent on wavelength, while in the absence of absorption it is a measure of the scattering and dependent on concentration and wavelength. In the presence of both absorption and scattering, the ratio defines a value for attenuation index at any given concentration. The attenuation index depends upon the cell length and the position of the cell insofar as they influence the fraction of total transmitted energy intercepted by the phototube in the measuring instrument.

\section{Correction for the Presence of Sucrose and Water}

In order to obtain $a_{c}^{*}$ due to the impurities of a commercial sugar solution, it is necessary to correct the observed transmittancy for the influence of sucrose and water. It is convenient to obtain the transmittancy of the solutions by using water in the reference cell of the spectrophotometer.

Sucrose solutions were intensively purified with adsorbents and the transmittancies determined. The purified sucrose solution was prepared as follows:

A sample of commercial granulated sugar was dissolved at $80^{\circ} \mathrm{C}$ in distilled water to 60.4 Brix $^{4}$ $(0.778 \mathrm{~g} / \mathrm{ml})$. The solution was treated four successive times with a washed activated carbon (15 $\mathrm{g}$ /liter of solution), stirred for $30 \mathrm{~min}$ at $80^{\circ} \mathrm{C}$, and filtered after each of the four treatments through an asbestos pad formed on No. 40 Whatman paper. After the last carbon treatment, the solution was filtered through a "fine" sintered glass filter. The $\mathrm{pH}$ was always kept between 6.8 and 7.2.

\footnotetext{
4 "Brix" is defined as the percentage by weight of dry sugar solids.
}

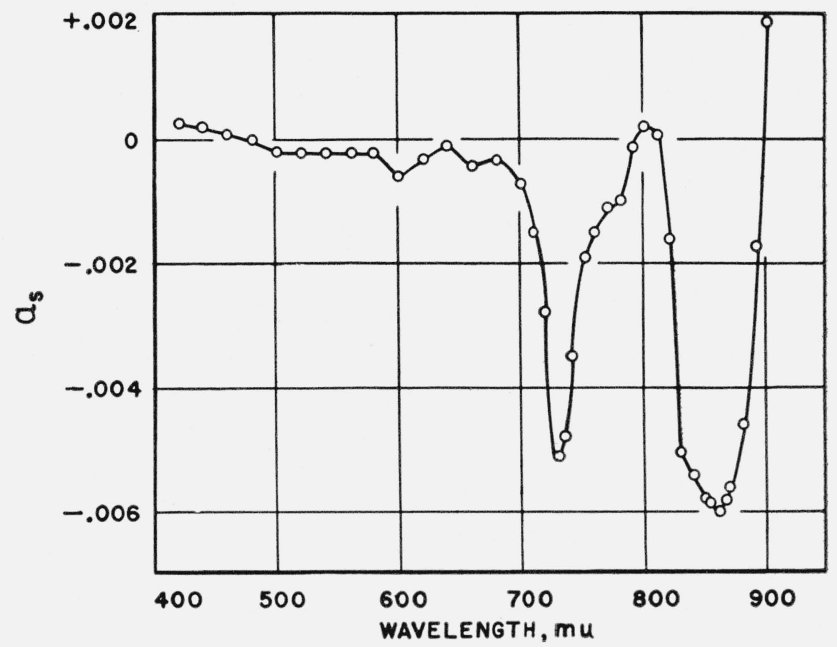

FiguRE 1. Dependence of $a_{8}$ upon wavelength for a purified sucrose solution $(0.778 \mathrm{~g} / \mathrm{ml})$ measured relative to distilled water a in 10-cm cell.

In figure $1, a_{e}^{*}$ for a sucrose solution $(0.778 \mathrm{~g} / \mathrm{ml})$ is plotted against the wavelength. The cell length was $10 \mathrm{~cm}$. It may be observed that $a_{c}^{*}$ is less than zero for wavelengths from 500 to $900 \mathrm{~m} \mu$, which is due to the fact that that reference cell contains more water than the sucrose solution. The minima observed at $740 \mathrm{~m} \mu$ and $860 \mathrm{~m} \mu$ coincide with harmonics in the infrared absorption spectrum of water [7].

The correction for sucrose and water has been applied at each wavelength to the data obtained for commercial sugar liquors. The correction for cell lengths of $1 \mathrm{~cm}$ or less has been found to be smaller than the uncertainties of the measurements in the visible spectrum. For very accurate work in cell lengths greater than $10 \mathrm{~cm}$, it is recommended that the correction for sucrose and water be determined in the instrument available for the work and at the particular concentrations and cell lengths desired.

\section{Experimental Results}

The sugar products investigated include Cuban and Hawaiian raws, washed Cuban raw, granulated sugars, invert mixtures, impure beet sugar, and blackstrap. The transmittancies were measured at wavelengths between 400 and $820 \mathrm{~m} \mu$ with a Beckman model DU spectrophotometer. The cell length was chosen so that the transmittancy readings were made in the range of 20 to 95 percent. These solutions were prepared by adding distilled water at room temperature to the raw sugar and warming the mixture with stirring to $80^{\circ} \mathrm{C}$. After straining through a 60 -mesh sieve, the cooled mixture was adjusted to a pH between 6.8 and 7.2 with a few drops of a dilute $\mathrm{NaOH}$ solution. The concentrations less than the maximum (about $0.75 \mathrm{~g} / \mathrm{ml}$ ) were obtained by dilution with water. The cell depth was $0.20 \mathrm{~cm}$. When the data are plotted in the conventional manner, that is, $-\log T_{s}$ against $c$, the devia- 

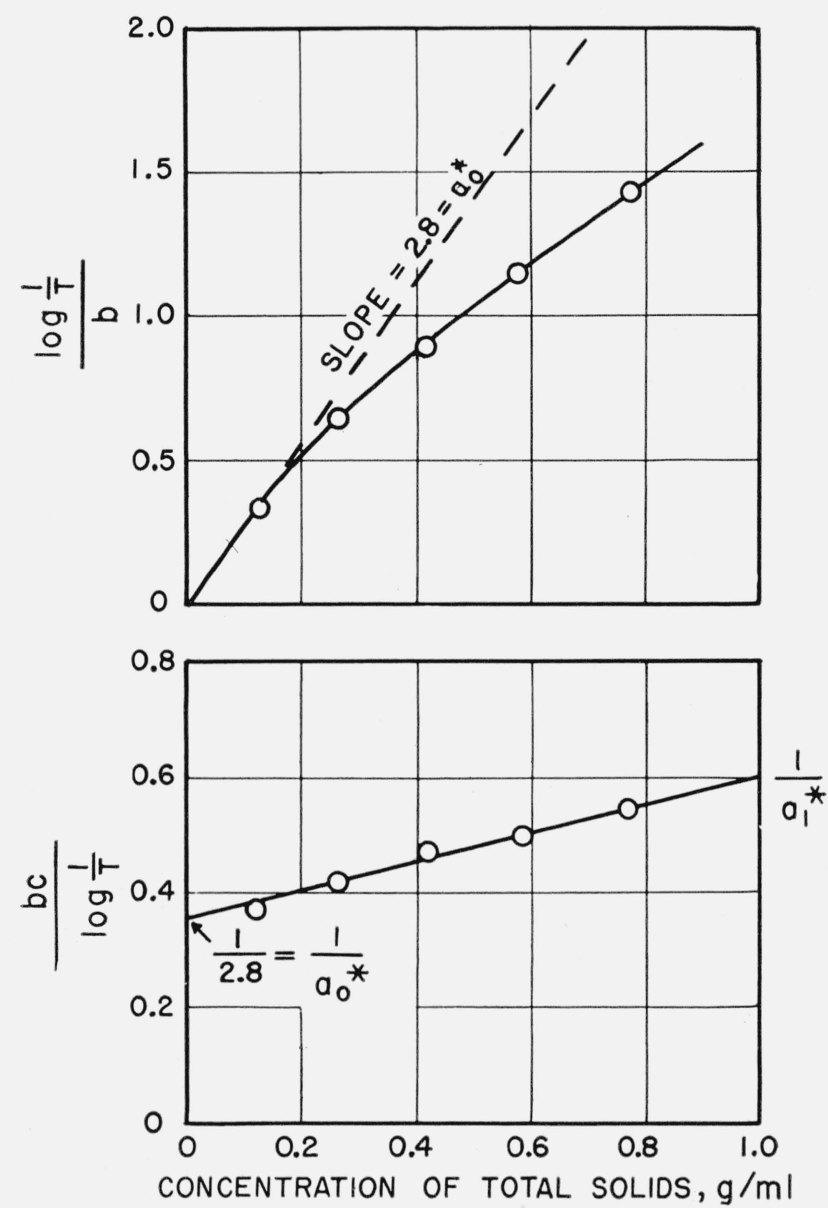

Figure 2. Relation between limiting slope of Beer's law and the intercept on a $1 / a_{c}^{*}$ versus c plot.

Data apply to an unfiltered Hawaiian raw sugar liquor at $550 \mathrm{~m} \mu$.

tion from Beer's law is quite evident. Figure 2 is such a plot of the data for the Hawaiian raw sugar.

It has been found that an accurate linear dependence on concentration is given by a plot of $1 / a_{c}^{*}$ against $c$. It is readily seen that the value for the limiting slope on the Beer's law plot shown at the top of figure 2 is equal to $a_{0}^{*}$, the reciprocal of the intercept. If $a_{1}^{*}$ denotes the attenuation index at $c=1$ and $a_{0}^{*}$ the value as the concentration approaches zero (infinite dilution), it is possible to define the straight line by the following relationship:

$$
\frac{1}{a_{c}^{*}}=\left[\frac{1}{a_{1}^{*}}-\frac{1}{a_{0}^{*}}\right] c+\left[\frac{1}{a_{0}^{*}}\right] \text {. }
$$

The slope is numerically equal to $\left[\left(1 / a_{1}^{*}\right)-\left(1 / a_{0}^{*}\right)\right]$ and the intercept is $\left(1 / a_{0}^{*}\right)$. However, the values of $a_{1}^{*}$ and $a_{0}^{*}$ can be more readily obtained from a graphical extrapolation.

Typical data are contained in figure 3 for the transmittances of unfiltered Hawaiian raw sugar solutions plotted according to eq (4). It is significant that the slope increased with increase in wavelength, but the reason for this is not evident. The increase in slope may depend upon the ratio of the absorption

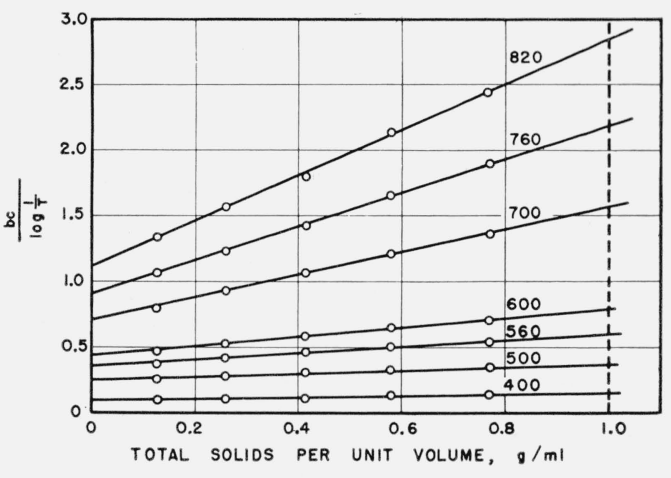

Figure 3. Unfiltered Hawaiian raw sugar observed in a cell length of $0.20 \mathrm{~cm}$ between wavelengths of 400 and $820 \mathrm{m \mu}$.

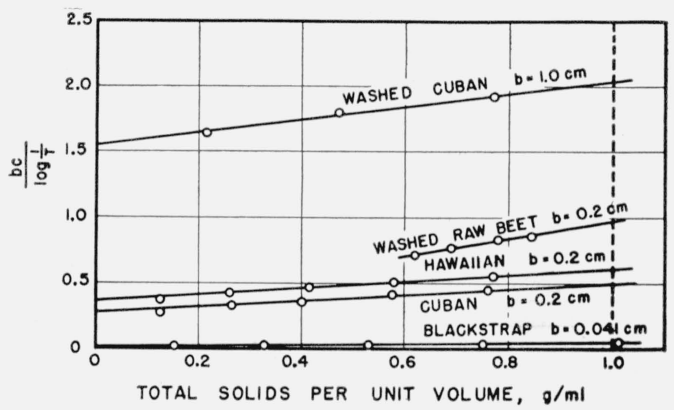

Figure 4. Linear plots for several raw sugars, impure beet sugar, and blackstrap observed at a wavelength of $560 \mathrm{m \mu}$.

to scattering at the various wavelengths. The slope has not been observed in any case to be negative.

The relationship given by eq (4) has been found to be valid for a large variety of sugar products. Typical data for some of these at $560 \mathrm{~m} \mu$ are given in figure 4. The washed Cuban raw was observed in a $1.00-\mathrm{cm}$ cell, the washed raw beet, Hawaiian raw, Cuban raw in $0.2-\mathrm{cm}$ cells, and the blackstrap in a $0.041-\mathrm{cm}$ cell.

The data for two unfiltered granulated sugar liquors are presented in figure 5. In these solutions the value of the attenuation index is quite small. Because of the relatively small quantity of absorbing nonsucrose solids, the small residual scattering in a granulated sugar solution contributes materially to the observed attenuation index. Some correction for scattering is necessary if a measure of the absorption alone is desired.

An obvious method of modifying the turbidity of sugar liquors is by high-speed centrifuging. A Cuban raw liquor was subjected to this treatment, and the results are of some interest. A gravitational field of 22,000 times gravity was obtained at an operating speed of $15,000 \mathrm{rpm}, 100,000$ times gravity at a speed of 40,000 rpm, and 150,000 times gravity at a speed of $50,000 \mathrm{rpm}$. All runs were made at substantially room temperature by means of special refrigeration of the equipment. ${ }^{5}$ Figure 6 is a plot of the resultant $\left[1 / a_{c}^{*}\right]$ versus $c$. After the centrifuging operation at

5 We are indebted to G. H. Hogeboom of the Cancer Research Section, National Institutes of Health, for the use of the ultracentrifuge equipment for attaining 100,000 times and 150,000 times gravity. 

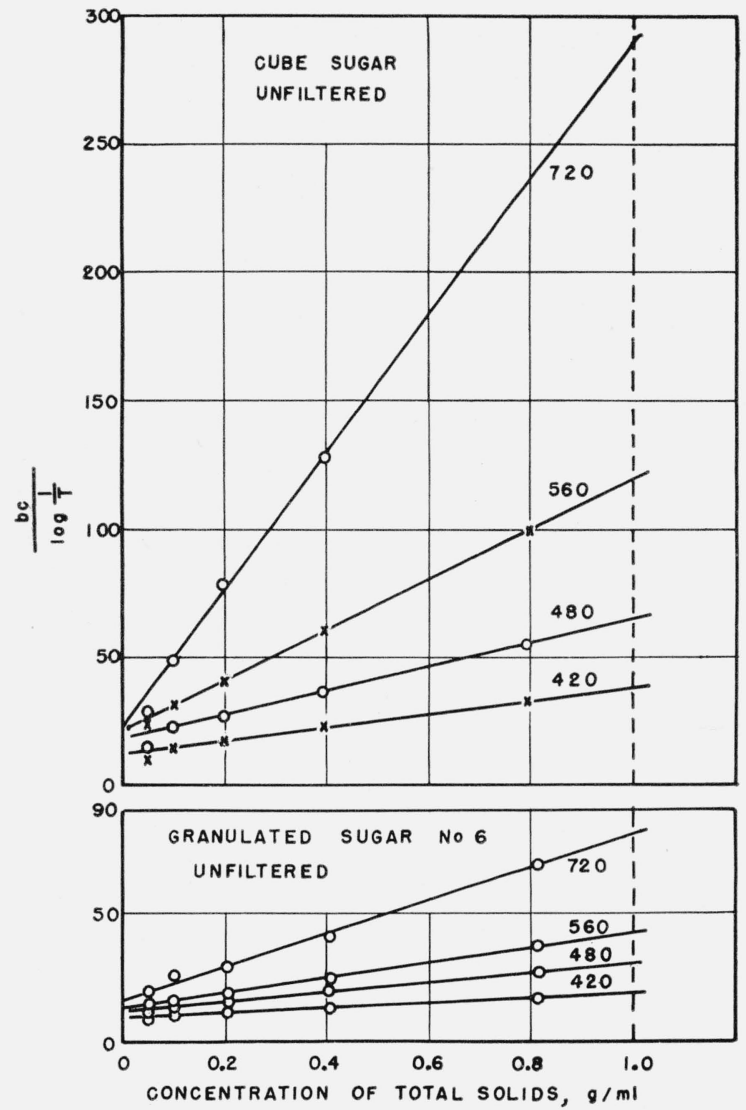

FIGURE 5. Linear plot of solutions of unfiltered granulated sugars at the wavelengths $(m \mu)$ indicated.

the highest gravity, a solid phase was observed at the surface of the liquor as well as at the bottom, which indicated a range of density of the suspended materials.

It may be seen from table 1 that the result of centrifuging the 60 Brix liquor at 22,000 times gravity was to decrease the attenuation indices $a_{0}^{*}$ and $a_{1}^{*}$. Apparently, the conditions of this experiment resulted in about the same percentage decrease in both $a_{1}^{*}$ and $a_{0}^{*}$. As the strength of the gravitational field increased, the values of $a_{1}^{*}$ and $a_{0}^{*}$ approached each other, indicating that the slope of the straight lines approach zero with increase in centrifuging. The values of $a_{0}^{*}$ and $a_{1}^{*}$ are influenced by time of centrifuging, gravity, and concentration of solution (which influences the viscosity of the solution) as is shown in table 1.

TABLE 1. Effects after centrifuging a Cuban raw liquor

\begin{tabular}{|c|c|c|c|}
\hline \multirow[b]{3}{*}{$\begin{array}{l}\text { Original unfiltered liquor } \\
22,000 \text { times gravith with } 60 \mathrm{Bx} \text { for } 30 \mathrm{~min} \\
22,000 \text { times gravity with } 40 \mathrm{Bx} \text { for } 30 \mathrm{~min} \\
100,000 \text { times gravity with } 40 \mathrm{~B} \text { for } 45 \mathrm{~min} \\
150,000 \text { times gravity with } 60 \mathrm{Bx} \text { for } 30 \mathrm{~min}\end{array}$} & \multirow{3}{*}{$\begin{array}{c}\begin{array}{c}\text { Cell } \\
\text { length }\end{array} \\
\mathrm{cm} \\
0.20 \\
.20 \\
.50 \\
.50 \\
.20\end{array}$} & \multicolumn{2}{|c|}{$\begin{array}{l}\text { Attenuation } \\
\text { index at } 560 \mathrm{~m} \mu\end{array}$} \\
\hline & & $a_{1}^{*}$ & $a_{0}^{*}$ \\
\hline & & $\begin{array}{l}2.10 \\
1.52 \\
\text { 1. } 30 \\
\text { 1. } 12 \\
1.34\end{array}$ & $\begin{array}{l}\text { 4. } 06 \\
2.45 \\
1.59 \\
1.23 \\
1.34\end{array}$ \\
\hline
\end{tabular}

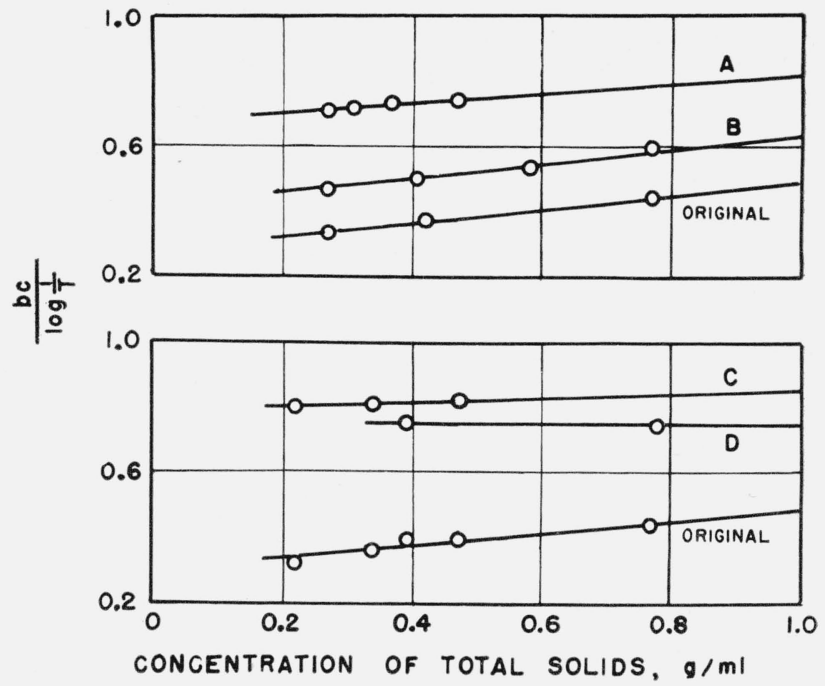

Figure 6. Effect of centrifuging a Cuban raw sugar.

Observations at $560 \mathrm{~m} \mu$ :

$\mathrm{A}$, Liquor at $40 \mathrm{Bx}$ for $30 \mathrm{~min}$ at 22,000 gravity; $\mathrm{B}$, liquor at $60 \mathrm{Bx}$ for $30 \mathrm{~min}$ at 22,000 gravity; C, liquor at $40 \mathrm{Bx}$ for 45 min at 100,000 gravity; D, liquor at $60 \mathrm{Bx}$ for $30 \mathrm{~min}$ at 150,000 gravity.

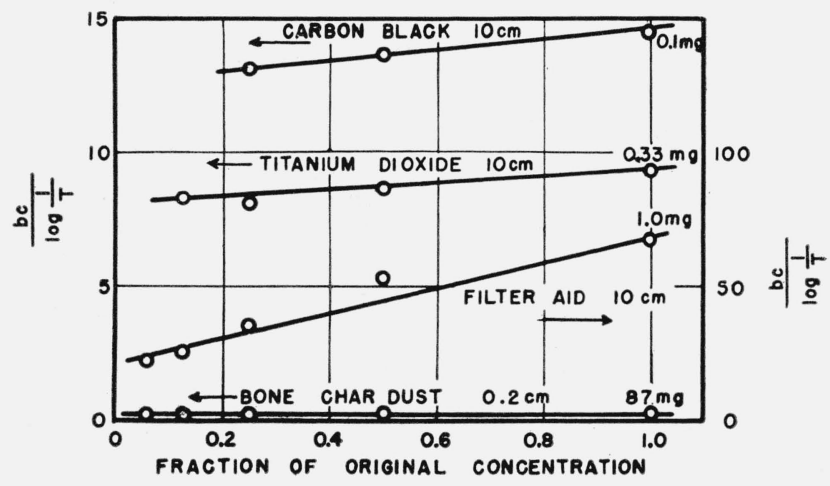

Figure 7. Linear plots for the addition of finely divided materials to a 60-Brix granulated-sugar liquor.

A series of measurements were made on solutions containing solids in suspension, and the results suggested that the linear dependence of $b c /-\log T_{s}$ on $c$ might be a general phenomenon. Figure 7 contains results for suspensions of a carbon black, titanium dioxide, a filter aid, and char dust in a 60 Brix granulated sugar solution. The cell length is indicated and the transmittances were measured at $560 \mathrm{~m} \mu$. The suspensions were prepared by adding each of the following materials to $30-\mathrm{g}$ portions of a 60 Brix liquor: carbon black $(0.1 \mathrm{mg})$, titanium dioxide $(0.33 \mathrm{mg})$, filter aid $(1.0 \mathrm{mg})$, and char dust $(87.0 \mathrm{mg})$. The dependence of $1 / a_{c}^{*}$ on $c$ was found to be linear, and this was also true for the same systems observed at other wavelengths. Similar results were obtained for titanium dioxide and graphite dispersed in glycerine and for powdered mica suspended in water. Calcium oxalate suspensions prepared by precipitation were also found to follow this relationship. 
It is obvious that in applications to turbidimetric analysis, it is not necessary that the suspension be sufficiently diluted to obey Beer's law. By using the method of plotting herein proposed, the concentration range of suspended material can be greatly increased for turbidimetric analysis.

\section{Remarks}

The linear dependence of $1 / a_{c}^{*}$ upon $c$ supplements the usual Beer's law considerations in dilute solutions. Transmittancy data on commercial sugar liquors can now be reported as attenuation indices at some reference concentration. It appears that either $a_{1}^{*}$ or $a_{0}^{*}$ can be used for this purpose.

Transmittancy values have long been used to evaluate the purifying effects of various adsorbents, such as bone char, on commercial sugar liquors. This necessitates observations on the sugar liquors before and after the treatment with the adsorbent. It is well to appreciate that the information obtained from such measurements is not the complete story in regard to the adsorbent problem. There may be present small yet significant impurities (for example, inorganic salts) that contribute little to the scattering and absorption in the visible range of wavelengths, but may contribute appreciably in ultraviolet regions. Such impurities may significantly influence the refining characteristics of the sugar liquor. However, the transmittancy measurements in the visible region serve a definite purpose, and they have the added advantage that the systems under study are not affected by the measurements.

\section{References}

[1] R. T. Balch and H. S. Paine, Colloid chemistry in the sugar industry, Colloid Chem. IV, p. 137-62 (1932) (edited by J. Alexander).

[2] F. W. Zerban, The color problem in sucrose manufacture, Technological Report No. 2, Sugar Research Foundation, Inc. (August 1947).

[3] K. S. Gibson, Spectrophotometry (200 to 1,000 millimicrons) NBS Circular 484 (1949).

[4] F. J. Bates and Associates, NBS Circular 440, p. 302 (1942).

[5] W. R. Brode, Chemical spectroscopy, p. 151 (John Wiley \& Sons, 2d ed., 1947).

[6] M. Halwer, J. Am. Chem. Soc. 70, 3985 (1948).

[7] N. E. Dorsey, Properties of ordinary water substance, Am. Chem. Soc. monograph No. 81 (1940).

Washington, September 19, 1952. 